\title{
Acknowledgement to Reviewers of Animals in 2017
}

\author{
Animals Editorial Office \\ MDPI AG, St. Alban-Anlage 66, 4052 Basel, Switzerland \\ Published: 9 January 2018
}

Peer review is an essential part in the publication process, ensuring that Animals maintains high quality standards for its published papers. In 2017, a total of 100 papers were published in the journal. Thanks to the cooperation of our reviewers, the median time to first decision was 23 days and the median time to publication was 64 days. The editors would like to express their sincere gratitude to the following reviewers for their time and dedication in 2017:

\author{
Adamczyk, Krzysztof \\ Allen, Kate \\ Allen, Michael \\ Ampe, Bart \\ Anagnostopoulos, Georgios \\ Andersen, Monica \\ Anderson, Kenneth \\ Aparicio, Miguel A. \\ Archer, Gregory S. \\ Arhant, Christine \\ Arkins, Sean \\ Arney, David \\ Ates, Serkan \\ Azarpajouh, Samaneh \\ Backus, Brittany L. \\ Bacon, Heather \\ Baker, Sandra \\ Baker, Kathy \\ Barbieri, Sara \\ Barnard, Shanis \\ Barnett, Mark \\ Battini, Monica \\ Bauer, Erika \\ Baumans, Vera \\ Beatty, Julia \\ Beck, Alan \\ Bennett, Elizabeth L. \\ Bentosela, Mariana \\ Berezowski, John \\ Berliner, Elizabeth \\ Biagi, Pier Francesco \\ Binanti, Diana \\ Bjalobok, Faith \\ Bland, Ian \\ Boe-Hansen, Gry
}

Bolea, Rosa

Bolwell, Charlotte

Boogaard, Birgit

Boone, John

Boyle, Laura

Brady, Colleen M.

Brearley, Jackie

Brook, Ryan

Broom, Donald

Buning, J.T. De Cock

Burghardt, Gordon

Bushby, Philip A.

Butterworth, Andrew

Büttner, Kathrin

Calambokidis, John

Cannon, Claire

Carney, Hazel C.

Carter, Anne

Cenci-Goga, Beniamino T.

Chandler, Cynthia Kay

Chastant-Maillard, Sylvie

Cheng, Heng-Wei

Chur-Hansen, Anna

Cirulli, Francesca

Citek, Jindrich

Clarke, Nancy

Cole, Matthew

Coombe, Jo

Corazzin, Mirco

Costa, Marcio

Costall, Alan

D'Occhio, Michael

Dai, Francesca

Dalla Villa, Paolo Felice

Dallaire, Jamie Ahloy 
D’Aniello, Biagio

Dashper, Kate

Davies, Anna

De Rensis, Fabio

Delgado, Mikel

Demyda-Peyrás, Sebastián

DeVries, Trevor

Di Giminiani, Pierpaolo

Dixon, Laura

Doneley, Bob

Donnett, Uri

Dórea, Fernanda

Doughty, Amanda

Doveren, Aldo

Dowsey, Andrew

Dunlop, Rebecca

Dvir, Hay

Dwyer, Cathy

Dzidic, Alen

Edinboro, Charlotte H.

Erasmus, Marisa A.

Errington, Timothy M.

Fàbrega, Emma

Farnworth, Mark J.

Fawcett, Anne

Fei, Andrew Chang-Young

Ferrante, Valentina

Fidani, Cristiano

Fine, Aubrey

Fishlock, Vicki

Font I Furnols, Maria

Franco, Nuno Henrique

Freeman, Lynetta

Freire, Raf

Galindo, Francisco

Gallis, Christos

Gamborg, Christian

Gardiner, Andrew

Garry, Franklyn

Gatel, Laure

Gebremedhin, Kifle

Geers, Rony

Georg, Heiko

Gianguzza, Paola

Giersberg, Mona Franziska

Gill, Margaret

Gillett, James

Gingerich, Eric

Gingrich, Elise N.

Glenk, Lisa Maria

Gold, Jenifer

Grajfoner, Dasha
Grandin, Temple

Grimm, Herwig

Grogono-Thomas, Rosemary

Guzek, Dominika

Ha, James C

Hadley, Phil

Hagen, Jenny

Hall, Sophie

Hall, Carol

Hanlon, Alison

Hargis, Billy

Hart, Benjamin

Harvey, Naomi

Hässig, Michael

Hassink, Jan

Haxel, Joe

Hazel, Susan

Heleski, Camie

Henry, Beverley

Hernandez, Carlos E.

Herskin, Mette S.

Hiby, Elly

Hickson, Rebecca

Hoffman, Christy

Hokkanen, Ann-Helena

Houpt, Katherine Albro

Howard, Veronica

Howell, Tiffani

Humer, Elke

Hunt, Melissa

Ijichi, Carrie

Irvine, Leslie

Izmirli, Serdar

Jacobs, Leonie

Johnson, Jay S.

Jonker, Arjan

Karsten, Cynthia

Kazdin, Alan

Keates, Helen

Kiddie, Jenna

Kjaer, Joergen

Knight, Andrew

Knight, Peter

Koluman-Darcan, Nazan

Kongara, Kavitha

Kortet, Raine

Krawczel, Peter D.

Kreisler, Rachael

Kuhar, Chris

Kurt, Timothy

Lassen, Jesper

Laurence, Michael 
Laven, Richard

Leach, Matthew

Lewis, Dave

Lopez-Salesansky, Noelia

Lovercamp, Kyle

MacKay, Jill

Macleod, Malcolm R.

Mahony, Timothy John

Main, David

Malashichev, Yegor

Maltz, Ephraim

Manning, Louise

Manteca, Xavier

Maple, Terry

Margerison, Jean

Marinelli, Lieta

Marsa-Sambola, Ferran

Martelli, Giovanna

Martin, Jessica

Matchock, Robert L.

Mather, Jennifer

Matsuoka, Atsuko

McGlone, John

McLennan, Krista

McMillin, Kenneth W.

Meagher, Rebecca

Meehan, Cheryl L.

Meijboom, Franck

Mellor, David

Mench, Joy

Mergenthaler, Marcus

Meyer, Iben

Meyer, Robert E.

Michalopoulou, Eleni

Michie, Craig

Miele, Mara

Molento, Carla Forte Maiolino

Montoya Alonso, Jose Alberto

Mooring, Mike

Morgan, Kathleen

Morgan-Davies, Clare

Morris, Kevin N.

Morton, David

Muboko, Never

Murison, Pamela

Nagy, Annamaria

Nannoni, Eleonora

Napolitano, Fabio

$\mathrm{Ng}$, Zenithson

Noonan, Michael

Oppedal, Frode

Osinga, Sjoukje A.
O'Sullivan, Siobhan

Panagiotopoulou, Olga

Paranhos Da Costa, Mateus José Rodrigues

Parkin, Tim

Pasicka, Edyta

Passantino, Annamaria

Patronek, Gary

Patterson, Paul

Pearce, Christopher

Pemberton, Neil

Peterson, Sam

Phalen, David

Piccolo, John

Pirrone, Federica

Plà, Lluis M.

Pound, Pandora

Progar, Amber Adams

Protopopova, Alexandra

Proudfoot, Kathryn

Quattrocchi, Fedora

Rast, Luzia

Rawlings, John Merritt

Reese, Laura

Reimert, Inonge

Ridoutt, Brad

Riley, Christopher

Rogers, Chris W.

Rogers, Lesley

Rollin, Bernard

Rowan, Andrew

Ruch Gallie, Rebecca

Ruhnke, Isabelle

Saleri, Roberta

Sant'Ana, Manuel Magalhães

Scheerlinck, Jean-Pierre

Schinckel, Allan P

Schmoelzl, Sabine

Schoster, Angelika

Schwarzer, Angela

Scobie, David

Scollo, Annalisa

Scullion Hall, Laura

Shaw, David

Shivley, Chelsey B.

Shriver, Adam

Siegford, Janice

Slater, Margaret R.

Smak, J.A.

Sneddon, Jennifer

Sparrey, Julian

Staaveren, Nienke Van

Stadig, Lisanne M. 
Stafleu, Frans

Statham, Poppy

Steibel, Juan Pedro

Stella, Judi

Stephenson, Mitchell B.

Stoeger, Angela

Sturaro, E.

Swinker, Ann M.

Szabo, Judit

Tadich, Tamara

Tan, Bie

Teague, Richard

Thornber, Peter

Thornton-Kurth, Kara

Tilbrook, Alan

Tiplady, Catherine

Todd, Teri

Toribio, Jenny-Ann

Tribe, Andrew

$\mathrm{Tu}$, Yan

Tucker, Corrina

Turner, Mark

Uwiera, Richard

Van Eerdenburg, Frank J C M

Van Heezik, Yolanda

Ventura, Beth

Verdon, Megan

Verstegen, Martin W. A.

Vevea, Jack

Von Essen, Erica

Voslarova, Eva
Waldmann, Karl Heinz

Walk, Carrie L.

Wallis, Lisa

Wang, K.

Watson, Andrea K.

Webster, John

Wellbrock, Wiebke

Wels, $\mathrm{H}$.

Weng, Hsin-Yi

Werner, Scott J.

White, Gavin

White, Steven

White, Rachel

White, Peter

Whiting, Terry

Whitley, David S.

Whittaker, Alexandra

Widmar, Nicole Olynk

Williams, Scott C.

Williams, Angelica

Wilson, David

Windschnurer, Ines

Winkel, Albert

Wohlfarth, Rainer

Wycislo, Kathryn

Wynne, Clive

Yamauchi, Hiroyuki

Yeates, James

Zaefarian, Fifi

Zervas, George

Zivotofsky, Ari

(C) 2018 by the authors. Submitted for possible open access publication under the terms and conditions of the Creative Commons Attribution (CC BY) license (http://creativecommons.org/licenses/by/4.0/). 